\title{
Protocol for an app-based affective control training for
}

\section{adolescents: proof-of-principle double-blind randomized}

\section{controlled trial [version 1; peer review: 3 approved, 1}

\section{approved with reservations]}

\author{
Susanne Schweizer (D1) Jovita T. Leung (D1), Rogier Kievit (D)2, \\ Maarten Speekenbrink ${ }^{3}$, William Trender (iD) 4, Adam Hampshire ${ }^{4}$, \\ Sarah-Jayne Blakemore(D1
}

\footnotetext{
${ }^{1}$ Institute of Cognitive Neuroscience, University College London, London, UK

${ }^{2}$ MRC Cognition and Brain Sciences Unit, University of Cambridge, Cambridge, UK

${ }^{3}$ Department of Experimental Psychology, University College London, London, UK

${ }^{4}$ Department of Medicine \& Centre for Neurotechnology Computational, Cognitive \& Clinical Neuroimaging Laboratory, Imperial Collge London, London, UK
}

V1 First published: 07 Jun 2019, 4:91
https://doi.org/10.12688/wellcomeopenres.15229.1

Latest published: 02 Oct 2019, 4:91

https://doi.org/10.12688/wellcomeopenres.15229.2

\begin{abstract}
Background: $75 \%$ of all mental health problems have their onset before the end of adolescence. Therefore, adolescence may be a particularly sensitive time period for preventing mental health problems. Affective control, the capacity to engage with goal relevant and inhibit distracting information in affective contexts, has been proposed as a potential target for prevention. In this study, we will explore the impact of improving adolescents' affective control capacity on their mental health.

Methods: The proof-of-principle double-blind randomized controlled trial will compare the effectiveness of an app-based affective control training (AC-Training) to a placebo training (P-Training) app. In total, 200 ( $50 \%$ females) adolescents (11-19 years) will train for 14 days on their training app. The AC-Training will include three different $n$-back tasks: visuospatial, auditory and dual (i.e., including both modalities). These tasks require participants to flexibly engage and disengage with affective and neutral stimuli (i.e., faces and words). The P-Training will present participants with a perceptual matching task. The three versions of the P-Training tasks vary in the stimuli included (i.e., shapes, words and faces). The two training groups will be compared on gains in affective control, mental health, emotion regulation and self-regulation, immediately after training, one month and one year after training.
\end{abstract}

Discussion: If, as predicted, the proposed study finds that AC-Training

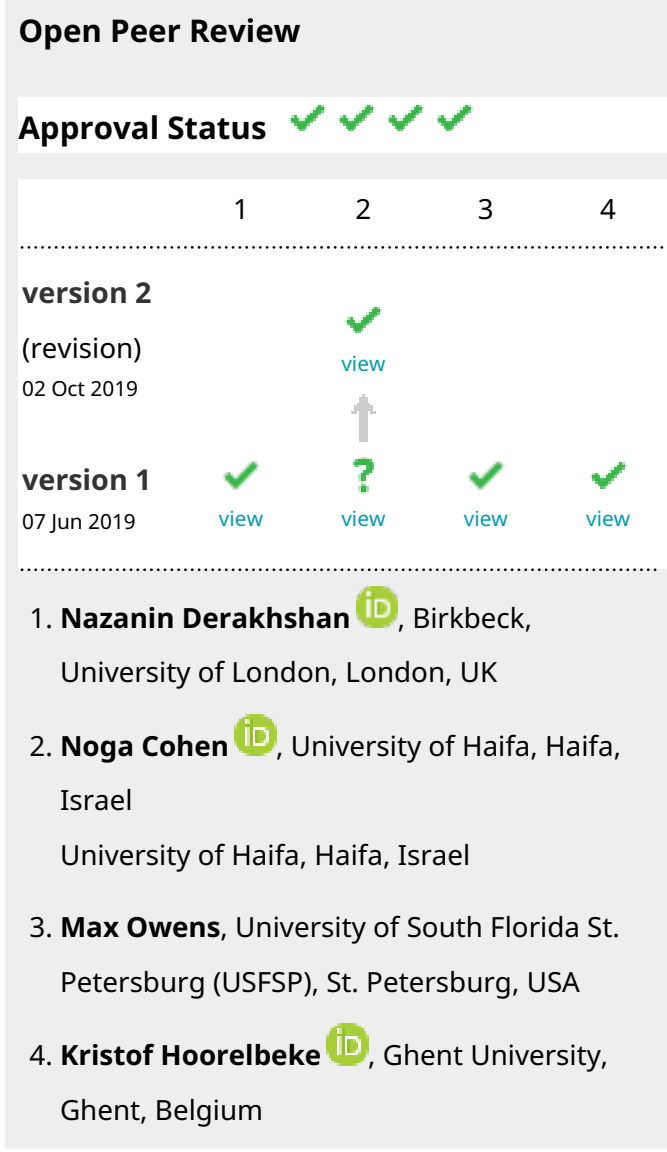


successfully improves affective control in adolescents, there would be significant potential benefits to adolescent mental health. As a free app, the training would also be scalable and easy to disseminate across a wide range of settings.

Trial registration: The trial was registered on December 10th 2018 with the International Standard Randomised Controlled Trial Number (Registration number: ISRCTN17213032).

\section{Keywords}

Mental health, Adolescence, Emotion regulation, Affective control, App-based training
Any reports and responses or comments on the article can be found at the end of the article.

Corresponding author: Susanne Schweizer (s.schweizer@ucl.ac.uk)

Author roles: Schweizer S: Conceptualization, Formal Analysis, Funding Acquisition, Investigation, Methodology, Project Administration, Resources, Supervision, Writing - Original Draft Preparation; Leung JT: Investigation, Project Administration; Kievit R: Data Curation, Formal Analysis, Methodology; Speekenbrink M: Data Curation, Formal Analysis; Trender W: Software; Hampshire A: Software;

Blakemore SJ: Conceptualization, Methodology, Supervision, Writing - Original Draft Preparation

Competing interests: $\mathrm{AH}$ is a co-founder of the company that developed the app software. He was not involved in task design nor will he be involved in any data analyses.

Grant information: This work was supported by the Wellcome Trust [209127; to SS], [107392; to RAK] and [104908 to SJB]; the MRC Programme [SUAG/014 RG91365; to RAK]; United Kingdom Research and Innovation (to SJB); and the Jacobs Foundation (to SJB). The funders had no role in study design, data collection and analysis, decision to publish, or preparation of the manuscript.

Copyright: ( 2019 Schweizer S et al. This is an open access article distributed under the terms of the Creative Commons Attribution License, which permits unrestricted use, distribution, and reproduction in any medium, provided the original work is properly cited.

How to cite this article: Schweizer S, Leung JT, Kievit R et al. Protocol for an app-based affective control training for adolescents: proof-of-principle double-blind randomized controlled trial [version 1; peer review: 3 approved, 1 approved with reservations] Wellcome Open Research 2019, 4:91 https://doi.org/10.12688/wellcomeopenres.15229.1

First published: 07 Jun 2019, 4:91 https://doi.org/10.12688/wellcomeopenres.15229.1 


\section{Abbreviations}

AC-Training = Affective control training; P-Training = Placebo training; DERS $=$ Difficulties in Emotion Regulation Scale

\section{Background}

$75 \%$ of all mental health problems have their onset before the end of adolescence ${ }^{1}$. Many of these disorders, for example major depressive disorder, will be recurrent throughout the lifespan creating large costs in human suffering ${ }^{2-4}$. Adolescence - here defined as starting with puberty and ending with the attainment of an independent adult role (10-24 years ${ }^{5}$ - thus may be a particularly sensitive time period for prevention of mental health problems $^{6}$. In this study, we will explore the impact of improving adolescents' affective control capacity on their mental health.

Affective control, the capacity to flexibly engage and disengage from affective information as required by current goal-demands, is impaired across a wide range of mental health problems? Poor affective control capacity has been shown to be associated with poor mental health outcomes over and above neutral 'cool' cognitive control during adolescence ${ }^{8-12}$. We have previously suggested that this association between affective control and mental health can be partially accounted for by emotion regulation ${ }^{12}$. That is, affective control constitutes the cognitive building blocks of successful emotion regulation. Emotion regulation refers to the automatic and volitional processes deployed to modify an individual's affective experiences ${ }^{13}$. Improving affective control in adolescents, whose everyday environments can include high levels of negative affect and affective fluctuations ${ }^{14-17}$, may then confer benefits to emotion regulation capacity and mental health.

Studies conducted in adults have shown that training affective control leads to improvements in both emotion regulation capacity and self-reported mental health ${ }^{18-21}$. Cool cognitive control training has also been shown to be effective in reducing symptoms of depression and anxiety, as well as improving emotion regulation $^{22-27}$.

The cognitive training literature in children and adolescents has largely focused on remediation for learning difficulties as well as neurodevelopmental disorders such as attention deficit and hyperactivity disorder $\left(\mathrm{ADHD}^{28,29}\right)$. Less is known about the impact of cognitive control training on young people's mental health ${ }^{30}$. A notable exception is the literature on cognitive training for adolescents with psychotic symptoms ${ }^{31,32}$, which synthesizing evidence suggests shows promising effects on symptoms and functioning ${ }^{33}$. However, given that affective control in particular may be impaired in adolescents with high levels of mental health problems, we will trial the effect of an affective control training (AC-Training) paradigm in our forthcoming study. Specifically, we will explore whether AC-Training improves adolescents' mental health and emotion regulation capacity and whether the magnitude of these improvements differs as a function of age.
The present study

The AC-Training that will be used in our study is a variant of a paradigm we applied successfully in adults and a preliminary study in adolescents with posttraumatic stress disorder ${ }^{21,34,35}$. The impact of the AC-Training will be assessed in a proofof-principle double-blind randomized controlled trial. In the AC-Training, participants will be presented with visuospatial, auditory and dual (combined visuospatial and auditory) versions of the $n$-back task. The three versions will, respectively, require participants to continuously update faces or words or both. On the first three days of the 14-day training programme, participants will train on one version each day. On days 4-14, participants are free to select any or all of the training tasks. However, they will be provided with a rationale suggesting that training on the dual $n$-back task is likely to confer more benefits than the other two versions. We hypothesise that opting to engage in a more challenging but potentially more beneficial task is an index of self-regulation, which has been shown to be associated with mental health across the lifespan ${ }^{36}$.

The tasks train affective control by requiring effective engagement and disengagement with affective information depending on task-demands. Affective valence is introduced to the training by including valenced stimuli. Specifically, the AC-Training will include $20 \%$ neutral, $20 \%$ positive and $60 \%$ negative stimuli. The rationale for including stimuli of different valences is that mental health problems can be characterised by difficulties disengaging from negative material ${ }^{37}$, avoidance of negative (e.g., threatening) information ${ }^{38}$ or aberrant processing of positive information ${ }^{39}$.

The effectiveness of the AC-Training will be compared with an active placebo training (P-Training), which includes three versions: shapes, words and faces from a feature match task. The P-Training requires participants to indicate whether the items presented in two panels are matched or mismatched. The training was designed to be minimally demanding on cognitive control, while exposing participants to the same stimuli as the AC-Training.

To investigate potential benefits of the AC-Training on mental health or emotion regulation, participants will complete selfreport measures immediately after training, after one month, and after one year. Additionally, the three facets of affective control - inhibiting attention and responses toward goal-irrelevant affective information, updating affective information or updating information in the context of affective distraction, and shifting flexibly between affective and non-affective task demands - will be assessed using experimental paradigms. Inhibition will be assessed with a modified version of Preston and Stansfield's ${ }^{40}$ affective Stroop task, which requires participants to categorize adjectives as either happy or sad that are superimposed over task-irrelevant faces that are either congruent, incongruent or neutral (i.e., scrambled). Updating will be assessed with the affective digit backward span task. In this task digits are serially 
presented over either a neutral or affective background image and then recalled in reverse order (modified version of standard digit span task; 41). Finally, shifting will be assessed with an affective card sorting task, which requires participants to flexibly switch between affective and neutral sorting rules ${ }^{12}$.

This study will allow us to investigate the following four hypotheses:

1. Affective control can be improved in adolescents (affective control training hypothesis). To investigate this hypothesis, we will compare individuals' performances on the affective $n$-back task, which is a slightly modified (i.e., including different stimuli and fewer trials) version of the AC-Training task, across the two training groups.

2. AC-Training compared to P-Training will lead to greater improvements in all facets of affective control as measured by non-trained affective control tasks, including affective inhibition, updating and shifting tasks (affective control facets hypothesis).

3. The benefits of AC-Training will decrease with age (age-related change hypothesis).

4. Increases in affective control from pre- to post-training will be associated with fewer self-reported mental health problems and emotion regulation difficulties, as well as higher levels of self-reported self-control, at each assessment time point (mental health hypothesis).

\section{Methods}

\section{Study setting}

The study will be run in schools in London, Cambridge and surrounding areas, and at the UCL Institute of Cognitive Neuroscience, UK.

\section{Participants}

In total, 200 adolescents ( 50\% female, 11-19 years) will be recruited through schools, advertisements on the lab website, the MQ research portal, the Anna Freud Centre "Schools in Mind" website, and social media. Recruitment will be stratified by age to ensure a proportional representation of each chronological year group. Including 200 participants (100 per training group) results in $\geq 93 \%$ power to detect an effect on our first hypothesis that affective control can be trained in adolescence. Power was established with time as within-subjects factor, training group as fixed factor and participants as random factors. The effect size for the interaction was estimated as small to medium $d=.40$, based on our previous training studies in adolescents $^{35}$ and adults ${ }^{21,34}$. The calculator used was https:// jakewestfall.shinyapps.io/pangea/.

Eligibility criteria. To be included participants will have to be between 11-19 years old and speak English fluently. Participants will be excluded from the study if they have a history of traumatic head injury, a diagnosed neurological or neurodevelopmental disorder, or if they are currently enrolled in another cognitive training intervention.
Allocation procedure. Included participants will be randomized to either the AC-Training or the P-Training groups. Condition allocation will be concealed to experimental staff by using computer-generated condition assignment (using Sealed Envelope simple randomisation service) stratified by age (young adolescents 11-14 years and mid-late adolescents 15-19 years; in line with: 12). Allocation will be based on a blocked randomization sequence with randomly mixed block sizes (2-6), which prevents the experimenter from deducing any potential sequencing even with awareness of the randomization type ${ }^{42}$. One experimenter (SS) will only conduct pre-training assessments and not be involved with any further participant testing as they will answer any queries about the training and technical issues that the participants may face during the training.

Blinding. For blinding procedures see extended data ${ }^{43}$. The procedures are uploaded to the trial registration page as a timestamped private document and will be made available online upon study completion. Following the final participant's followup assessment (one year after the second testing session, T2), all participants will receive an email describing the study purpose and giving them access to all training tasks for 12 months.

\section{Training procedure and timeline}

Participants complete a pre-training assessment, followed by 14 days of training within a four-week period. Within one week of the end of the four-week period, participants will complete the post-training assessment. Any deviations from the per protocol timeline due to the constraints of school-based testing will be accounted for in our analyses (see Statistical analyses section). Thirty days after the post-training assessment, participants will be asked to complete an online follow-up assessment. A final follow-up assessment will be completed one year after the post-training session. For a schematic overview of the study timeline see Figure 1.

Training phase. During the training phase, participants from both training groups will be presented with three different training tasks (see below for descriptions). On the first three days they will complete a different version of the training task each day. The presentation order of the three versions will be fixed for days 1-3. The P-Training group will complete the shapes (A), words (B), and faces (C) versions on the first, second, and third day of training, respectively. The AC-Training group will complete the visuospatial (A), auditory (B), and dual (i.e., including both modalities; C) versions of the training task on the first three days of training. From the fourth day of training onward participants in both groups will be free to select any of the three different versions of their training schedule.

At the beginning of the training, both groups will be told that they should spend as much time as possible training on version $\mathrm{C}$ due to its benefits to attention, memory and emotion regulation. Version $\mathrm{C}$ in the AC-training will be significantly more cognitively demanding ${ }^{44}$ than $\mathrm{A}$ and $\mathrm{B}$, whereas there are no differences in cognitive demands between versions $\mathrm{A}, \mathrm{B}$ or $\mathrm{C}$ in the P-Training. Emulating the design of established measures of academic diligence and self-regulation ${ }^{45-47}$, the ratio of time 


\begin{tabular}{|c|c|c|c|c|c|c|c|}
\hline \multirow{2}{*}{ Pre-training assessment } & \multicolumn{4}{|c|}{ Training phase } & \multirow{2}{*}{ Post-training assessment } & \multirow{2}{*}{$\begin{array}{l}\text { 1-month online follow-up } \\
\text { assessment }\end{array}$} & \multirow{2}{*}{$\begin{array}{c}\text { 1-year online follow-up } \\
\text { assessment }\end{array}$} \\
\hline & A & B & C & $\mathrm{A} / \mathrm{B} / \mathrm{C}$ & & & \\
\hline \multirow[t]{2}{*}{$\mathrm{T} 1$} & & & & & T2 & T3 & T4 \\
\hline & $\begin{array}{l}\text { Start } \\
\text { End } 0\end{array}$ & $\begin{array}{l}\text { ate } n \\
\text { ate w }\end{array}$ & $\begin{array}{l}\text { thin } \\
\text { hin } 4\end{array}$ & $\begin{array}{l}\text { veek of } T 1 \\
\text { eeks of } T 1\end{array}$ & $\begin{array}{l}\text { Within } 7 \text { days of the } \\
\text { last day of training }\end{array}$ & 30 days after $\mathrm{T} 2$ & 1 year after $\mathrm{T} 2$ \\
\hline
\end{tabular}

Figure 1. Study timeline. $T 1-T 4=$ Assessment time point $1-4 ; A / B / C=$ refers to the three different versions of the training tasks available in both training groups; Pre- and post-training assessment = Assessment sessions run prior to and after completing the training phase; Training phase = period of training on the app; 1-month and 1-year online follow up assessment = key outcomes will be assessed online one month and one year following the completion of the training.

spent training on version $\mathrm{C}$ relative to $\mathrm{A}$ and $\mathrm{B}$ will be taken as a behavioural index of self-regulation.

Procedure on each training day. On each training day tasks will be populated with a different set of stimuli. In both training groups participants will be given the option to end the training any time from 10 mins onward. The full training session will take between 20-30 min depending on the level achieved. There will be no limit on the number of training sessions they can complete during a day. Training sessions that are less than 10 mins will not be considered as full training sessions, and will not be included in the analyses nor will participants be compensated for these sessions.

Each time they start the training, participants will be asked four brief questions about their mood, affect regulatory intentions, social context and current activity. To assess current mood, participants will be asked, "How happy do you feel right now?". They will provide their mood rating by moving the cursor on a visual analogue scale ranging from "Very unhappy" to "Very happy". Affect regulatory intentions will be assessed with the question, "Are you trying to change the way you feel right now?". They will be offered nine answer options from a dropdown menu. Participants will be able to select "No." or "Yes, by ..." followed by different types of regulatory strategies (i.e., distraction, problem-solving, behavioural activation, reappraisal, avoidance, social support, acceptance or other). Social context will be assessed by selecting from a dropdown menu to indicate whether right now they are: "Alone", "With others (friends/family)", or "With others (strangers)." Finally, participants will indicate their current activity from a selection of eleven options on a dropdown menu (e.g., commuting, school/ work).

Adherence/retention. Participants will be compensated for each section of the study to incentivise enrolment and study completion. They will be paid $£ 10$ for each pre- and post-training assessments (T1 \& T2) and $£ 5$ for both the online follow-up assessments (T3 \& T4). Participants will additionally receive $£ 2$ per completed training day. If participants complete two or more sessions on a single day they will be paid $£ 5$.

Training. In addition to payments, retention will be optimised by sending participants a daily training reminder at $8 \mathrm{am}$.
Participants who have not completed at least 10 mins of training by $5 \mathrm{pm}$ will be sent an additional reminder, informing them that they have a training session waiting for them. A final reminder will be sent at $8 \mathrm{pm}$ for any participants who have not completed their minimum training requirement by then.

Follow-up. Two weeks after the initial request to complete the follow-up assessments, email reminders will be sent to incentivise follow-up completion. Reminder emails will be sent at weekly intervals, until the follow-up assessments are completed or until the maximum number of reminders (i.e., three) has been sent, whichever comes first.

\section{Training tasks}

Affective control training. The three versions of the ACtraining tasks are described below and depicted in Figure 2.

Training progression. The first three days will start at $n=1$. From day 4 onward participants will select one of the versions to train on and training will start at the average level of $n$-back achieved on the previous training session with the selected version. During each individual training session, the difficulty level will be titrated to each participant's maximum capacity with $n$ increasing by 1 if performance reaches $\geq 70 \%$ accuracy and decreasing by 1 when accuracy is $\leq 30 \%$. Accuracy feedback will be provided after each response. A red boarder will flash up around the grid for false alarms (participant presses Match on a non-target trial) or misses (participant presses No Match on a target trial or fails to provide any response). A green boarder will flash up for all correctly classified trials.

Stimuli. Each of the training versions will include $20 \%$ neutral stimuli and $80 \%$ affective stimuli to train the flexible engagement and disengagement from affective information. $30 \%$ of the trials will constitute target trials. The words included in the dual and auditory versions of the AC-Training are derived from the Affective Norms for English Words database ${ }^{48}$ and with the exception of the positive words were included in previous versions of this training $\operatorname{task}^{21,34}$. Positive stimuli are included in the current training task because of the salience of positive material in adolescence ${ }^{49}$, as well as research showing a critical role of reward processing in the onset of mental health problems $^{50,51}$. The words are $20 \%$ neutral, $30 \%$ positive and $50 \%$ negative. 


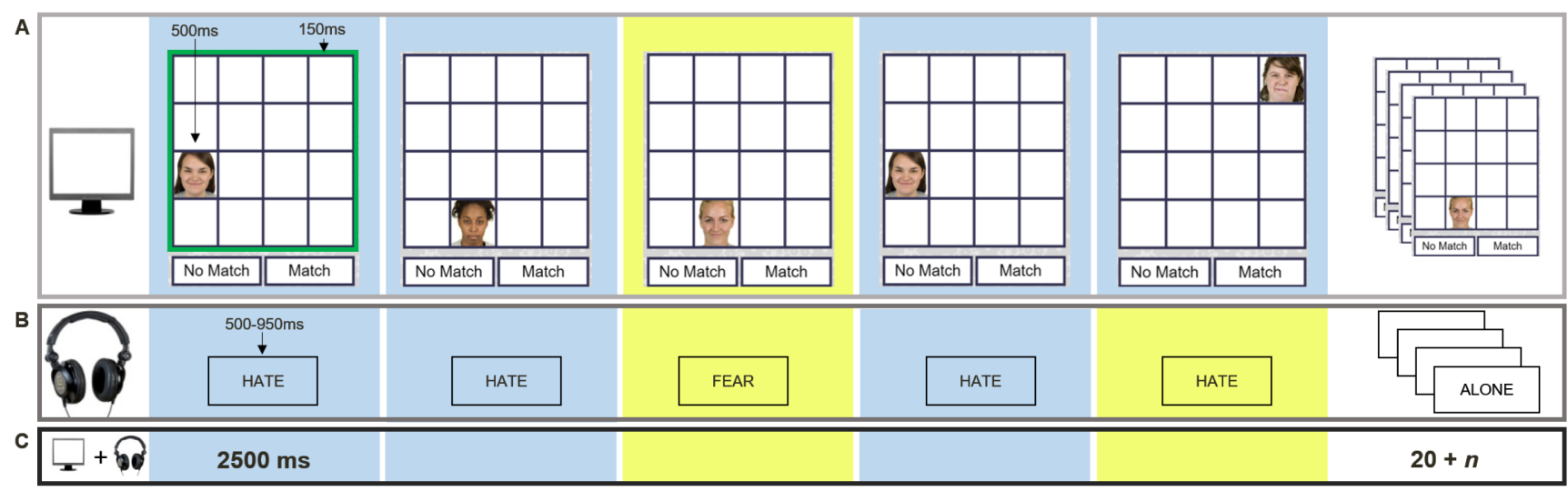

Figure 2. Affective control training tasks. The figure depicts sample trials for each of the three training tasks: A) visuospatial $n$-back, B) auditory $n$-back, and C) dual $n$-back task. Trials depicted with a light blue background require a "No Match" button press, whereas yellow backgrounds indicate "Match" (i.e., target) trials in the respective modality. The green border provides feedback to participants, where green indicates the response was correct, whereas a red border appears for incorrect trials. Feedback is provided after each response or when a trial times out. The example block in Figure 2 is depicted for $n=1$. Match trials for the visuospatial $n$-back training task are trials where the current face is presented in the same location as the face $n$ positions back. For auditory $n$-back match trials, the same word is presented as the one $n$ trials back. The dual $n$-back training task includes both modalities and both types of target trials (for additional buttons appearing on screen with the dual $n$-back see the task description below). $2500 \mathrm{~ms}=$ the maximal (duration is self-paced up to $2500 \mathrm{~ms}$ ) time between onset of one stimulus and the next (i.e., total trial time); $500 \mathrm{~ms}=$ face presentation time; $150 \mathrm{~ms}=$ feedback presentation time; $500-950 \mathrm{~ms}=$ word presentation time. $20+n=$ each block consists of $20+n$ trials.

The faces stimuli were selected from several different databases, which are licenced for use online, to provide a diverse stimulus set in terms of demographics and emotional expressions. The databases included are: the Chicago Face Database ${ }^{52}$, the Radboud Faces Database ${ }^{53}$, the London Face Research Set ${ }^{54}$, the Emotional Faces Stimulus Set ${ }^{55}$, and the NIMH Child Emotional Faces Picture Set ${ }^{56}$. Our final stimulus set includes child, adolescent and adult faces of female and male gender. The ethnic appearances of the faces included are African, Asian, Caucasian, Latin American, Mediterranean, Middle Eastern, and Mixed Race. We denote the ethnicity here as appearance only as not all the actors' origins were recorded across the different databases. The emotional expression of the faces included are happy, angry, fearful, sad, and neutral. In each training session $50 \%$ of the faces are female, $50 \%$ of the faces are from child and adolescent models. The affective expressions included in each training session are $20 \%$ neutral, $20 \%$ angry, $20 \%$ fearful, $20 \%$ sad (i.e., $60 \%$ negative), $20 \%$ happy.

Visuospatial n-back. In the visuospatial $n$-back task faces appear for $500 \mathrm{~ms}$ on a $4 \times 4$ grid. The task requires participants to indicate within $2.5 \mathrm{~s}$ whether the face they are seeing in the current trial is presented in the same location as the face presented $n$ trials back. Responses are provided via "No Match" or "Match" button press.

Auditory $\boldsymbol{n}$-back. In the auditory version of the training task participants are presented with words over headphones. On each trial they have $2.5 \mathrm{~s}$ to indicate via button press (see visuospatial $n$-back), whether the word presented in the current trial is the same as they heard $n$ trials back.
Dual $\boldsymbol{n}$-back. The dual version of the task presents participants with the visuospatial and auditory $n$-back simultaneously. The task requires participants to indicate whether the location in which the face is appearing on the current trial is the same as the location in which a face appeared $n$ trials back. At the same time, they indicate whether the word they are hearing on the current trial is the same as the word $n$-trials back. The response options include four buttons: "No Match" for non-target trials, "Location Match", for trials including only a visuospatial target, "Word Match", for trials including only an auditory target, and "Both Match" for trials including both an auditory and visuospatial target. One third of the target trials are visuospatial targets, one third auditory targets, and one third dual targets.

Placebo training. The P-Training task requires participants to indicate via button press ("Match", "No Match") whether two panels display exactly the same stimuli in the same positions on a grid. In the shapes version the stimuli are random geometric shapes. The faces and words versions include the same stimuli as the AC-Training. Each trial is self-timed up to a maximum of $90 \mathrm{~s}$ after which participants are asked to respond more quickly.

\section{Pre- and post-training session assessments}

For an overview of all measures that will be included in T1-T4 see Figure 3.

Demographics. Self-identified gender, ethnicity and parental education level will be assessed. Parental education will be included as a proxy measure for socioeconomic status (SES). 


\begin{tabular}{|c|c|c|c|c|c|c|}
\hline & Enrolment & Allocation & $\mathrm{T} 1$ & $\mathrm{~T} 2$ & $\mathrm{~T} 3$ & $\mathrm{~T} 4$ \\
\hline \multicolumn{7}{|l|}{ Participant characteristics } \\
\hline Inclusion \& exclusion criteria & $\mathrm{X}$ & & & & & \\
\hline Allocation & & $\mathrm{X}$ & & & & \\
\hline Age & & $X$ & $\mathrm{X}$ & & & \\
\hline Gender & & & $\mathrm{X}$ & & & \\
\hline Parental education (SES) & & & $\mathrm{X}$ & & & \\
\hline Pubertal development & & & $\mathrm{X}$ & & & \\
\hline \multicolumn{7}{|l|}{ Mood \& mental-health } \\
\hline Positive and Negative Affect Scale & & & $\mathrm{X}$ & $\mathrm{X}$ & $\mathrm{X}$ & $\mathrm{X}$ \\
\hline Strength and Difficulties Questionnaire & & & $\mathrm{X}$ & $\mathrm{X}$ & $\mathrm{X}$ & $\mathrm{X}$ \\
\hline Difficulties in Emotion Regulation Scale & & & $\mathrm{X}$ & $\mathrm{X}$ & $\mathrm{X}$ & $\mathrm{X}$ \\
\hline Brief Self-Control Scale & & & $\mathrm{X}$ & $\mathrm{X}$ & $\mathrm{X}$ & $\mathrm{X}$ \\
\hline \multirow{2}{*}{\multicolumn{7}{|c|}{$\begin{array}{l}\text { Experimental measures of affective and cognitive } \\
\text { functioning }\end{array}$}} \\
\hline & & & & & & \\
\hline Affective backward digit span task & & & $\mathrm{X}$ & $\mathrm{X}$ & & \\
\hline Affective set-shifting task & & & $\mathrm{X}$ & $\mathrm{X}$ & & \\
\hline Affective Stroop task & & & $\mathrm{X}$ & $\mathrm{X}$ & & \\
\hline Fluid intelligence & & & $\mathrm{X}$ & & & \\
\hline \multicolumn{7}{|l|}{ Training tasks } \\
\hline Benchmark visuospatial $n$-back task & & & $\mathrm{X}$ & $\mathrm{X}$ & & \\
\hline Benchmark feature match (placebo) task & & & $\mathrm{X}$ & $\mathrm{X}$ & & \\
\hline \multicolumn{7}{|l|}{ Training } \\
\hline Affective Control Training & & & $\bullet$ & $\rightarrow$ & & \\
\hline Placebo Control Training & & & $\leftrightarrow$ & 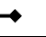 & & \\
\hline
\end{tabular}

Figure 3. Schedule of enrolment, interventions and assessments (SPIRIT). SPIRIT = Standard Protocol Items: Recommendations for Interventional Trials. $\mathrm{T} 1$ = pre-training assessment; $\mathrm{T} 2$ = post-training assessment; $\mathrm{T} 3=1$-month follow-up assessment; $\mathrm{T} 4=1$-year follow-up assessment.

Parental education has been shown to be a robust indicator of $\mathrm{SES}^{57}$ and has been previously used by our group in similar samples (e.g., 58).

Pubertal development. Pubertal development will be assessed with the well-validated, self-report Pubertal Development Scale ${ }^{59}$. The scale will be sent to participants via email link, so that they can complete it in private at home.

Self-reported mood and mental health. All self-report measures will be administered on a computer screen.

Positive and negative affect. To assess current positive and negative affect participants will complete the state version of the Positive and Negative Affect Schedule ${ }^{60}$. The scale requires individuals to rate the extent to which 10 positive and 10 negative adjectives describe them. We will ask participants to rate the adjectives with respect to how well they describe them over the past week.

Mental health difficulties. Mental health problems will be assessed with the Strengths and Difficulties questionnaire. The questionnaire is a 25 -item self-report measure, which is divided into five subscales ${ }^{61,62}$. Four of the subscales measure difficulties and one subscale measures a strength, prosocial behaviour. The difficulties subscales assess emotional symptoms (internalizing symptoms), conduct problems, hyperactivity/inattention and peer relationship problems. The measure has been shown to have good psychometric properties in the age group that will be recruited for the current study (Cronbach's $\alpha$ of 0.80 ), as well as good sensitivity, specificity and prospective utility ${ }^{63-65}$.
Emotion regulation. The 36-item Difficulties in Emotion Regulation Scale (DERS) will be administered to assess emotion regulation ${ }^{66}$. The DERS has six subscales that measure: nonacceptance, the propensity to experience secondary negative emotions in response to negative emotions; goals, difficulties engaging with goal-directed behaviours when upset; impulse, the ability to control one's behaviour when experiencing negative emotions; awareness, the tendency to attend to emotions; strategies, individuals' perception that emotions cannot be controlled; and clarity; individuals' ability to correctly identify their emotions ${ }^{66}$. The scale has shown high internal consistency, Cronbach's $\alpha=0.93^{66}$ and has been reliably used in the age range included in the current study ${ }^{67}$.

Self-regulation. The Brief Version ${ }^{68}$ of the Self-Control Scale ${ }^{69}$ will be administered to measure self-regulation. The scale has 13 items and has shown good internal consistency ${ }^{68,70}$,

Cognitive-affective task performance. Three tasks will be included to assess the impact of AC-Training relative to P-Training on the different facets of affective control: inhibition, updating and set-shifting.

Inhibition. Inhibition of affective interference will be assessed using an affective Stroop task ${ }^{40}$. In this modified version of the task participants indicate whether adjectives are happy or sad. The words are superimposed on the image of a face, resulting in three trial types: congruent (emotions of the word and face are matched), incongruent (emotions of the word and face are mismatched) or neutral (the word is superimposed on scrambled face image). This modified version of the task 
includes only happy and sad as emotion categories, whereas the original task also included words and faces expressing anger. The current version also includes only four words per emotion category and the faces are from two adult actors and two child/ adolescent actors (50\% female). These modifications were made to adapt the difficulty level of the task for younger participants and to make the stimuli age-appropriate. The face stimuli are derived from the same face databases as the training stimuli.

Feedback is provided after each trial with a red or green border appearing around the image for $200 \mathrm{~ms}$, indicating an error or correct response, respectively. Trials are self-paced up to $4 \mathrm{~s}$. If no response is detected a red border appears and the next trial is presented. There are 96 trials in total with each actor being paired with each of the eight adjectives in each condition.

Shifting. The capacity to shift flexibly between task-demands will be assessed using an affective set-shifting task. The task is an affective version of the Madrid Card Sorting Task ${ }^{71}$. Participants are dealt a card, which they are asked to assign to one of four decks according to three possible sorting rules: card color, number of items and shape (neutral version) or emotional expression (affective version). Sorting rule switch randomly after 6 to 9 trials (on average after 8 trials). Each rule is presented twice in the neutral and affective versions each, resulting in 96 trials. Participants are required to respond within $30 \mathrm{~s}$, after which the trials are recorded as an error. The presentation order of the affective and neutral versions is randomized. Performance on the task is operationalized as random errors. These are errors that occur on any trial in the series after the initial two trials (needed to establish the correct sorting rule). Random errors are most reliably associated with mental health outcomes in adolescents on this version of the task ${ }^{12}$.

Updating. Updating will be assessed with an affective backward digit span task, where participants are presented with digits $(1500 \mathrm{~ms})$ in serial order. The task starts with two digits per trial. Following the final digit in each trial, a keypad appears and participants are required to enter the digits in reverse order. Each span level is presented twice on this task. At least one out of two correct trials per span level is needed for progression to the next level. If both trials are incorrect the task is terminated. To manipulate valence, the digits are presented over negative and neutral background images. The images are from the Geneva Affective Picture Database ${ }^{72}$.

Fluid intelligence. The 12-item version of the Raven's Advanced Progressive Matrices will be used to assess intelligence ${ }^{73}$. Participants will be told that they should complete the task as quickly as possible. The measure has good psychometric properties $^{74}$. We chose the Raven's Advanced Progressive Matrices because it is sensitive in the wide age range included in the current study.

Benchmark training tasks. The benchmark versions of the training tasks will be identical to the training versions with a few exceptions noted below.
Visuospatial n-back tasks. The benchmark version of ACTraining is identical to the visuospatial $n$-back that will be used in the AC-Training with the exception that in the benchmarking version of the task only four blocks will be presented. Two of the blocks will include faces and two blocks will include scrambled faces.

Placebo task. For the benchmark version of the P-Training we will present the faces version of the feature match task. The version is identical to the training version with the exception that it is only $90 \mathrm{~s}$ long.

\section{Data management}

Following study completion all data will be linked-anonymized, with the linking documents being kept on separate encrypted drives. Fully anonymized data will be made open access through managed open access following the publication of our findings. That is, any researcher will be provided with our data if they consent to adhere to the General Data Protection Regulation and the British Psychological Society's Code of Ethics and Conduct. Our consent procedure will inform participants of these data storage and sharing procedures.

\section{Statistical analyses}

Statistical analyses will be performed using $\mathrm{R}^{75}$. Prior to all hypotheses testing, the two training groups will be compared on age using a Bayesian $t$-test to ensure that stratification was successful. The groups will then be compared on the following potential confounds using non-parametric Chi-square tests for binary and general linear modelling for continuous variables: gender, parental education, pubertal stage, intelligence, time interval between pre- and post-training (days); testing location and testing groups size to experimenter ratio. Any variables showing significant group differences at baseline will be added to all subsequent group comparisons as covariate.

Next, we will explore the structure of our outcome measures of interest at baseline. Specifically, we will explore the structure of affective and cognitive functioning using structural equation modelling (SEM). We hypothesize that cognitive control is best modelled using separate factors for affective, versus neutral, item content, such that the model in Figure 4 will outperform a single-factor model.

To investigate the first hypothesis (affective control training hypothesis) we will use mixed effects modelling with training group as fixed effect and time as within-subject effect. The outcome of interest will be $d$ ' achieved on the affective $n$-back task. Additionally, we will explore the impact of training on reaction time as a secondary outcome of interest. For the secondary analyses to be considered significant, we will apply a Bonferroni correction to reduce the threshold for statistical significance for two comparisons (accuracy and reaction) to $\alpha \leq .025$. We will then explore whether any effects of group and time are moderated by total training time (mins), total number of individual training sessions or ratio of time spent training on training task $\mathrm{C}$ relative to $\mathrm{A}$ and $\mathrm{B}$. We plan to include these overlapping measures separately as they arguably provide 


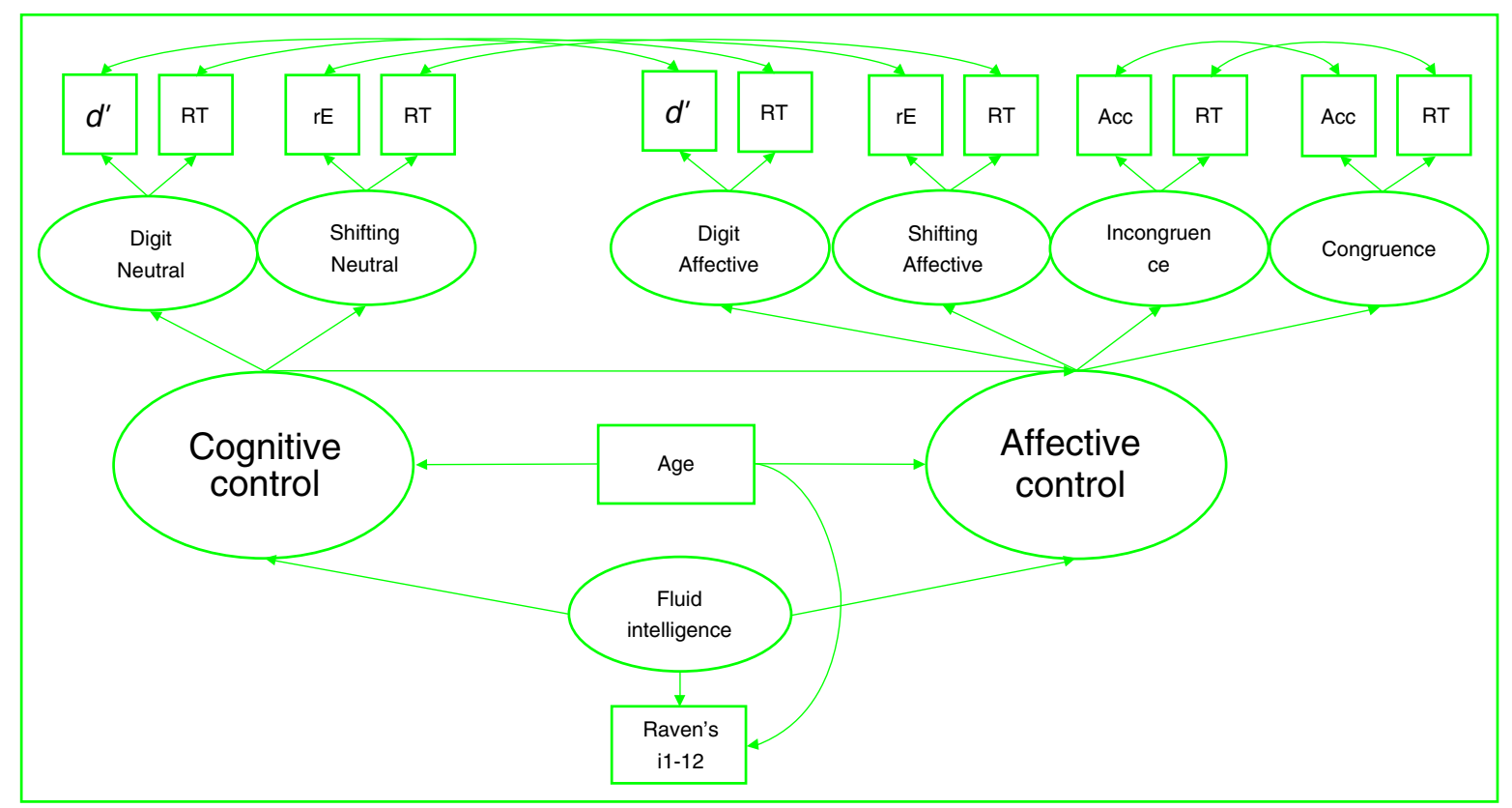

Figure 4. Predicted structure of affective and cognitive functioning at baseline. The figure offers a schematic representation of the predicted structure of cognitive and affective control in adolescents. Raven's i1 - $12=$ items $1-12$ on the Raven's Advanced Progressive Matrices; d' = d prime on backward digit span; RT = reaction time; rE = proportion random errors; Acc = percentage trials correct. Rectangular boxes = measured variables; ovals = latent constructs.

different types of information. Specifically, total training time will allow us to explore dose-response relationships. The other two measures, we propose, index more motivational factors such as diligence and/or motivation by the monetary incentive. To facilitate interpretation of any potential moderating effect, we will use a SEM trees approach to these moderators and enter the relevant groupings as moderators.

Our second hypothesis (affective control facets hypothesis), that AC-Training will improve inhibition, updating and shifting, will be tested with a multivariate mixed effects model. Time and group will be included as fixed effects and the three measures of affective control as outcomes of interest (i.e., working memory updating, inhibition, and set-shifting). The primary outcome of interest is accuracy, as this has been shown to be a more sensitive than reaction time in dissociating between individuals with and without mental health problems ${ }^{7}$. As with the first hypothesis, we will investigate whether any training benefits are moderated by total training time (mins), total number of individual training sessions or time spent training on task $\mathrm{C}$ relative to $\mathrm{A}$ and $\mathrm{B}$. The analyses will be repeated with reaction time as a secondary outcome of interest.

Our age-related change hypothesis, will be tested by including age a moderator in the multivariate mixed effects model used to test the affective control training hypothesis. The potentially moderating effect of age will be investigated using SEM trees. SEM trees identify age groupings to the benefits conferred by training. While the exploration is data-driven, it is theoretically informed by the literature showing differential effects of cognitive training on young compared to older adolescents ${ }^{76}$. A second exploratory analysis will include age as a continuous variable in the same model to investigate any linear or polynomial effects of age.

Fourth, to test the mental health hypothesis, we will use latent change score models, a subclass of SEM approaches ${ }^{77}$, which naturally allows for the integration of predictors of rates of change (e.g. improvements in mental health). Specifically, we will investigate whether pre- to post-training changes in the affective control factor established at baseline are associated with fewer self-reported mental health problems and emotion regulation difficulties, as well as higher levels of self-reported selfcontrol at each assessment time point. The primary analyses will include the post-training and one-month follow-up assessment. Secondary analyses will include the one-year follow-up.

\section{Research ethics approval}

Ethical approval for this study has been conferred by the University College London (UCL) Research Ethics Committee on 23 April 2018; Project ID:12753/002. Any protocol amendments will be submitted to the UCL Research Ethics Committee Chair for approval and recorded on the Open Science Framework pre-registration documentation.

\section{Consent and assent procedures}

Assent and consent will be obtained from prospective participants and their legal guardians, respectively. For participants under 18 years, study information and consent forms will first be sent to the parents. Parents will then have the opportunity to read the information and contact the research team with any potential questions. Children of parents who provide consent 
will be asked to provide written informed assent. Participants aged 18 years and over will be asked to provide written informed consent. When participants are tested at the pre- and post-training assessments they will be reminded that they can withdraw consent at any time before, during or after the study without any consequence and that they will be compensated for any part of the study completed until withdrawal.

\section{Availability of data}

Consent from participants will be obtained to share data through managed access. Researchers wishing to access the data need to consent to storing and analyzing the data in line with the General Data Protection Regulations and the British Psychological Society's Code of Ethics and Conduct.

\section{Dissemination policy}

The study has a multi-component dissemination policy: academic, stake-holders and public.

Academic. Standard academic dissemination of the study results will be sought through journal publications. Findings will also be communicated at scientific conferences and where permitted by journal regulations published on pre-print archives.

Stakeholders. Findings will be communicated via email to all research participants in a newsletter style communication. The main trial outcome paper will also be submitted as a Frontiers for Young Minds article and if accepted sent to all participants. We will further present the findings during a school talk in any of the participating schools that are interested in this option.

Public. The findings will also be communicated to the public by presenting them at public talks as well as through social media and, if interest can be generated, conventional broadcast or print media.

\section{Trial status}

The trial data collection started 21 September 2018 and the funding end date for this trial is 08 January 2022. Pre-training assessments have been completed in 64 participants, but none of these participants have completed training or any post-training assessments. This is protocol version 1 (30 November 2018).

\section{Conclusions}

If, as predicted, the proposed study finds that AC-Training successfully improves affective control in adolescents, there would be significant potential benefits to adolescent mental health. As a free app, the training would also be scalable and easy to disseminate across a wide range of settings.

\section{Data availability}

Underlying data

No data are association with this article.

\section{Extended data}

Open Science Framework: Protocol for an App-Based Affective Control Training for Adolescents. https://doi.org/10.17605/OSF. $\mathrm{IO} / 6 \mathrm{THSN}^{43}$

This project contains the following extended data:

- Supplementary Materials - WOR.pdf (Additional methods to main manuscript and completed SPIRIT checklist)

\section{Reporting guidelines}

Open Science Framework: SPIRIT checklist for Protocol for an App-Based Affective Control Training for Adolescents. https://doi.org/10.17605/OSF.IO/6THSN ${ }^{43}$

Data are available under the terms of the Creative Commons Zero "No rights reserved" data waiver (CCO 1.0 Public domain dedication).

\section{Grant information}

This work was supported by the Wellcome Trust [209127; to SS], [107392; to RAK] and [104908 to SJB]; the MRC Programme [SUAG/014 RG91365; to RAK]; United Kingdom Research and Innovation (to SJB); and the Jacobs Foundation (to SJB).

\section{Acknowledgements}

The authors would like to thank all the adolescents, teachers and parents that already have and those that will consent to participating in this study.
1. Kessler RC, Chiu WT, Demler O, et al:: Prevalence, severity, and comorbidity of 12-month DSM-IV disorders in the National Comorbidity Survey Replication. Arch Gen Psychiatry. 2005; 62(6): 617-27. PubMed Abstract | Publisher Full Text | Free Full Text

2. Breslau J, Gilman SE, Stein BD, et al.: Sex differences in recent first-onset depression in an epidemiological sample of adolescents. Transl Psychiatry. 2017; 7(5): e1139.

PubMed Abstract | Publisher Full Text | Free Full Text

3. Kovacs M, Obrosky S, George C: The course of major depressive disorder from childhood to young adulthood: Recovery and recurrence in a longitudinal observational study. J Affect Disord. 2016; 203: 374-81. PubMed Abstract | Publisher Full Text | Free Full Text
4. Wilson S, Vaidyanathan U, Miller MB, et al.: Premorbid risk factors for major depressive disorder: are they associated with early onset and recurrent course? Dev Psychopathol. 2014; 26 (4 Pt 2): 1477-93. PubMed Abstract | Publisher Full Text | Free Full Text

5. Sawyer SM, Azzopardi PS, Wickremarathne D, et al:: The age of adolescence. Lancet Child Adolesc Health. 2018; 2(3): 223-8.

PubMed Abstract | Publisher Full Text

6. Lee FS, Heimer H, Giedd JN, et al:: Mental health. Adolescent mental health-opportunity and obligation. Science. 2014; 346(6209): 547-9. PubMed Abstract | Publisher Full Text | Free Full Text

7. Schweizer S, Satpute AB, Atzil S, et al:: The impact of affective information on working memory: A pair of meta-analytic reviews of behavioral and 
neuroimaging evidence. Psychol Bull. 2019.

Publisher Full Text

8. Cohen-Gilbert JE, Thomas KM: Inhibitory control during emotional distraction across adolescence and early adulthood. Child Dev. 2013; 84(6): 1954-66. PubMed Abstract | Publisher Full Text | Free Full Text

9. Davidovich S, Collishaw S, Thapar AK, et al.: Do better executive functions buffer the effect of current parental depression on adolescent depressive symptoms? J Affect Disord. 2016; 199: 54-64. PubMed Abstract | Publisher Full Text | Free Full Text

10. Hare TA, Casey BJ: The neurobiology and development of cognitive and affective control. Cogn Brain Behav. 2005; 9(3): 273-286. Reference Source

11. Kilford EJ, Foulkes L, Potter R, et al:: Affective bias and current, past and future adolescent depression: a familial high risk study. J Affect Disord. 2015; 174: 265-71. PubMed Abstract | Publisher Full Text | Free Full Text

12. Schweizer S, Parker J, Leung JT, et al:: Age-related differences in affective control and its association with mental health difficulties. Dev Psychopathol. 2019; 1-13.

PubMed Abstract | Publisher Full Text

13. Gross JJ: Emotion regulation: affective, cognitive, and social consequences. Psychophysiology. 2002; 39(3): 281-91. PubMed Abstract | Publisher Full Tex

14. Klimstra TA, Kuppens P, Luyckx K, et al.: Daily Dynamics of Adolescent Mood and Identity. J Res Adolesc. 2015; 26(3): 459-73.

PubMed Abstract | Publisher Full Text

15. Larson RW, Moneta G, Richards MH, et al.: Continuity, stability, and change in daily emotional experience across adolescence. Child Dev. 2002; 73(4): 1151-1165. PubMed Abstract | Publisher Full Text

16. Maciejewski DF, van Lier PA, Branje SJ, et al:: A 5-Year Longitudinal Study on Mood Variability Across Adolescence Using Daily Diaries. Child Dev. 2015; 86(6): 1908-21.

PubMed Abstract | Publisher Full Text

17. Riediger M, Klipker K: Emotion regulation in adolescence. In: Handbook of emotion regulation. 2nd ed. New York, N.Y.: Guilford Press; 2015; 187-202. Reference Source

18. Cohen $\mathrm{N}$, Mor $\mathrm{N}$ : Enhancing reappraisal by linking cognitive control and emotion. Clin Psychol Sci. 2018; 6(1): 155-63.

Publisher Full Text

19. Cohen N, Mor N, Henik A: Linking executive control and emotional response a training procedure to reduce rumination. Clin Psychol Sci. 2015; 3(1): 15-25. Publisher Full Text

20. Cohen $\mathrm{N}$, Ochsner KN: From surviving to thriving in the face of threats: the emerging science of emotion regulation training. Curr Opin Behav Sci. 2018; 24: 143-155.

Publisher Full Text

21. Schweizer S, Grahn J, Hampshire A, et al.: Training the emotional brain: improving affective control through emotional working memory training. J Neurosci. 2013; 33(12): 5301-11.

PubMed Abstract | Publisher Full Text

22. Cohen N, Margulies DS, Ashkenazi S, et al.: Using executive control training to suppress amygdala reactivity to aversive information. Neuroimage. 2016; 125 : 1022-31.

PubMed Abstract | Publisher Full Text

23. Course-Choi J, Saville H, Derakshan N: The effects of adaptive working memory training and mindfulness meditation training on processing efficiency and worry in high worriers. Behav Res Ther. 2017; 89: 1-13.

PubMed Abstract | Publisher Full Text

24. Keshavan MS, Vinogradov S, Rumsey J, et al:: Cognitive training in mental disorders: update and future directions. Am J Psychiatry. 2014; 171(5): 510-522. PubMed Abstract | Publisher Full Text | Free Full Text

25. Koster EHW, Hoorelbeke K, Onraedt T, et al:: Cognitive control interventions for depression: A systematic review of findings from training studies. Clin Psychol Rev. 2017: 53: 79-92.

PubMed Abstract | Publisher Full Text

26. Motter JN, Devanand DP, Doraiswamy PM, et al.: Computerized Cognitive Training for Major Depressive Disorder: What's Next? Front Psychiatry. 2015; 6: 137. PubMed Abstract | Publisher Full Text | Free Full Text

27. Xiu L, Zhou R, Jiang $\mathrm{Y}$ : Working memory training improves emotion regulation ability: Evidence from HRV. Physiol Behav. 2016; 155: 25-29. PubMed Abstract | Publisher Full Tex

28. Cortese S, Ferrin M, Brandeis D, et al.: Cognitive training for attention-deficit/ hyperactivity disorder: meta-analysis of clinical and neuropsychological outcomes from randomized controlled trials. J Am Acad Child Adolesc Psychiatry. 2015; 54(3): 164-74.

PubMed Abstract | Publisher Full Text | Free Full Text

29. Kirk HE, Gray K, Riby DM, et al:: Cognitive training as a resolution for early executive function difficulties in children with intellectual disabilities. Res Dev Disabil. 2015; 38: 145-60.

PubMed Abstract | Publisher Full Tex

30. Hollis C, Falconer CJ, Martin JL, et al:: Annual Research Review: Digital health interventions for children and young people with mental health problems systematic and meta-review. J Child Psychol Psychiatry. 2017; 58(4): 474-503. PubMed Abstract | Publisher Full Tex
31. Choi J, Corcoran CM, Fiszdon JM, et al:: Pupillometer-based neurofeedback cognitive training to improve processing speed and social functioning in individuals at clinical high risk for psychosis. Psychiatr Rehabil J. 2017; 40(1) 33-42.

PubMed Abstract | Publisher Full Text | Free Full Text

32. Loewy R, Fisher M, Schlosser DA, et al:: Intensive Auditory Cognitive Training Improves Verbal Memory in Adolescents and Young Adults at Clinical High Risk for Psychosis. Schizophr Bull. 2016; 42 suppl 1: S118-S126. PubMed Abstract | Publisher Full Text | Free Full Text

33. Revell ER, Neill JC, Harte M, et al:: A systematic review and meta-analysis of cognitive remediation in early schizophrenia. Schizophr Res. 2015; 168(1-2): cognitive PubMed Abstract | Publisher Full Text

34. Schweizer S, Hampshire A, Dalgleish T: Extending brain-training to the affective domain: increasing cognitive and affective executive control through emotional working memory training. PLoS One. 2011; 6(9): e24372. PubMed Abstract | Publisher Full Text | Free Full Text

35. Schweizer S, Samimi Z, Hasani J, et al.: Improving cognitive control in adolescents with post-traumatic stress disorder (PTSD). Behav Res The. 2017: 93: 88-94.

PubMed Abstract | Publisher Full Text | Free Full Text

36. Heatherton TF, Wagner DD: Cognitive neuroscience of self-regulation failure. Trends Cogn Sci. 2011; 15(3): 132-9.

PubMed Abstract | Publisher Full Text | Free Full Text

37. Koster EHW, De Lissnyder E, Derakshan N, et al.: Understanding depressive rumination from a cognitive science perspective: the impaired disengagement hypothesis. Clin Psychol Rev. 2011; 31(1): 138-45.

PubMed Abstract | Publisher Full Text

38. Bar-Haim Y, Lamy D, Pergamin L, et al.: Threat-related attentional bias in anxious and nonanxious individuals: a meta-analytic study. Psychol Bull. 2007; 133(1): 1-24.

PubMed Abstract | Publisher Full Text

39. Admon R, Pizzagalli DA: Dysfunctional Reward Processing in Depression. Curr Opin Psychol. 2015; 4: 114-8.

PubMed Abstract | Publisher Full Text | Free Full Text

40. Preston SD, Stansfield RB: I know how you feel: task-irrelevant facial expressions are spontaneously processed at a semantic level. Cogn Affect Behav Neurosci. 2008; 8(1): 54-64.

PubMed Abstract | Publisher Full Text

41. Lezak MD: Neuropsychological assessment. New York, N.Y.: Oxford University Press; 1995

Reference Source

42. Suresh K: An overview of randomization techniques: An unbiased assessment of outcome in clinical research. J Hum Reprod Sci. 2011; 4(1): 8-11. PubMed Abstract | Publisher Full Text | Free Full Text

43. Schweizer S, Leung JT, Kievit R, et al.: Protocol for an App-Based Affective Control Training for Adolescents. 2019. http://www.doi.org/10.17605/OSF.IO/6THSN

44. Loosli SV, Buschkuehl M, Perrig WJ, et al.: Working memory training improves reading processes in typically developing children. Child Neuropsychol. 2012; 18(1): 62-78.

PubMed Abstract | Publisher Full Text

45. Duckworth AL, Grant H, Loew B, et al.: Self-regulation strategies improve selfdiscipline in adolescents: benefits of mental contrasting and implementation intentions. Educ Psychol. 2011; 31(1): 17-26.

Publisher Full Text

46. Galla BM, Plummer BD, White RE, et al.: The Academic Diligence Task (ADT): Assessing Individual Differences in Effort on Tedious but Important Schoolwork. Contemp Educ Psychol. 2014; 39(4): 314-25.

PubMed Abstract | Publisher Full Text | Free Full Text

47. Yeager DS, Henderson MD, Paunesku D, et al.: Boring but important: a selftranscendent purpose for learning fosters academic self-regulation. $J$ Pers Soc Psychol. 2014; 107(4): 559-80.

PubMed Abstract | Publisher Full Text | Free Full Text

48. Bradley BP, Lang PJ: Affective Norms for English Words (ANEW): Instruction manual and affective ratings. Technical report C-1, Gainesville, FL.: The Center for Research Psychophysiology. University of Florida; 1999. Reference Source

49. Foulkes L, Blakemore SJ: Is there heightened sensitivity to social reward in adolescence? Curr Opin Neurobiol. 2016; 40: 81-5.

PubMed Abstract | Publisher Full Tex

50. Forbes EE, Dahl RE: Research Review: altered reward function in adolescent depression: what, when and how? J Child Psychol Psychiatry. 2012; 53(1): 3-15. PubMed Abstract | Publisher Full Text | Free Full Text

51. Luking KR, Pagliaccio D, Luby JL, et al.: Reward Processing and Risk for Depression Across Development. Trends Cogn Sci. 2016; 20(6): 456-68. PubMed Abstract | Publisher Full Text | Free Full Text

52. Ma DS, Correll J, Wittenbrink B: The Chicago face database: A free stimulus set of faces and norming data. Behav Res Methods. 2015; 47(4): 1122-35. PubMed Abstract | Publisher Full Text

53. Langner O, Dotsch R, Bijlstra G, et al:: Presentation and validation of the Radboud Faces Database. Cogn Emot. 2010; 24(8): 1377-1388. Publisher Full Text 
54. DeBruine L, Jones B: Face Research Lab London Set. 2017. Reference Source

55. Meuwissen AS, Anderson JE, Zelazo PD: The creation and validation of the Developmental Emotional Faces Stimulus Set. Behav Res Methods. 2017; 49(3): 960-6. PubMed Abstract | Publisher Full Text | Free Full Text

56. Egger HL, Pine DS, Nelson E, et al.: The NIMH Child Emotional Faces Picture Set (NIMH-ChEFS): a new set of children's facial emotion stimuli. Int J Methods Psychiatr Res. 2011; 20(3): 145-56. PubMed Abstract | Publisher Full Text | Free Full Text

57. Dubow EF, Boxer P, Huesmann LR: Long-term Effects of Parents' Education on Children's Educational and Occupational Success: Mediation by Family Interactions, Child Aggression, and Teenage Aspirations. Merrill Palmer $Q$ (Wayne State Univ Press). 2009; 55(3): 224-49. PubMed Abstract | Publisher Full Text | Free Full Text

58. Fuhrmann D, Schweizer S, Leung J, et al.: The neurocognitive correlates of academic diligence in adolescent girls. Cogn Neurosci. 2019; 10(3): 88-99. PubMed Abstract | Publisher Full Text | Free Full Text

59. Petersen AC, Crockett L, Richards M, et al.: A self-report measure of pubertal status: Reliability, validity, and initial norms. J Youth Adolesc. 1988; 17(2): 117-33.

PubMed Abstract | Publisher Full Text

60. Watson D, Clark LA, Tellegen A: Development and validation of brief measures of positive and negative affect: the PANAS scales. J Pers Soc Psychol. 1988; 54(6): 1063-70. PubMed Abstract | Publisher Full Text

61. Goodman R: The Strengths and Difficulties Questionnaire: a research note. J Child Psychol Psychiatry. 1997; 38(5): 581-6. PubMed Abstract | Publisher Full Text

62. Goodman R, Ford T, Simmons H, et al:: Using the Strengths and Difficulties Questionnaire (SDQ) to screen for child psychiatric disorders in a community sample. Br J Psychiatry. 2000; 177(6): 534-9. PubMed Abstract | Publisher Full Text

63. Becker A, Rothenberger A, Sohn A, et al:: Six years ahead: a longitudinal analysis regarding course and predictive value of the Strengths and Difficulties Questionnaire (SDQ) in children and adolescents. Eur Child Adolesc Psychiatry. 2015; 24(6): 715-25. PubMed Abstract | Publisher Full Text

64. Goodman A, Goodman R: Population mean scores predict child mental disorder rates: validating SDQ prevalence estimators in Britain. $J$ Child Psychol Psychiatry. 2011; 52(1): 100-8. PubMed Abstract | Publisher Full Text

65. Goodman R: Psychometric properties of the strengths and difficulties questionnaire. J Am Acad Child Adolesc Psychiatry. 2001; 40(11): 1337-45. PubMed Abstract | Publisher Full Text
66. Gratz KL, Roemer L: Multidimensional assessment of emotion regulation and dysregulation: Development, factor structure, and initial validation of the difficulties in emotion regulation scale. J Psychopathol Behav Assess. 2004; 26(1): 41-54.

Publisher Full Text

67. Neumann A, van Lier PA, Gratz KL, et al:: Multidimensional assessment of emotion regulation difficulties in adolescents using the Difficulties in Emotion Regulation Scale. Assessment. 2010; 17(1): 138-149. PubMed Abstract | Publisher Full Text

68. Finkenauer C, Engels R, Baumeister R: Parenting behaviour and adolescent behavioural and emotional problems: The role of self-control. Int J Behav Dev. 2005; 29(1): 58-69. Publisher Full Text

69. Tangney JP, Baumeister RF, Boone AL: High self-control predicts good adjustment, less pathology, better grades, and interpersonal success. $J$ Pers. 2004; 72(2): 271-324.

PubMed Abstract | Publisher Full Text

70. Righetti F, Finkenauer C: If you are able to control yourself, I will trust you: the role of perceived self-control in interpersonal trust. J Pers Soc Psychol. 2011; 100(5): 874-86.

PubMed Abstract | Publisher Full Text

71. Barceló F: The Madrid card sorting test (MCST): a task switching paradigm to study executive attention with event-related potentials. Brain Res Brain Res Protoc. 2003; 11(1): 27-37.

PubMed Abstract | Publisher Full Text

72. Dan-Glauser ES, Scherer KR: The Geneva affective picture database (GAPED): a new 730-picture database focusing on valence and normative significance. Behav Res Methods. 2011; 43(2): 468-77. PubMed Abstract | Publisher Full Text

73. Raven JC: Guide to the standard progressive matrices. London: HK Lewis; 1960. Reference Source

74. Raven J: The Raven's progressive matrices: change and stability over culture and time. Cogn Psychol. 2000; 41(1): 1-48.

PubMed Abstract | Publisher Full Text

75. R Core Team: R: A language and environment for statistical computing R Foundation for Statistical Computing; 2013. Reference Source

76. Knoll LJ, Fuhrmann D, Sakhardande AL, et al:: A Window of Opportunity for Cognitive Training in Adolescence. Psychol Sci. 2016; 27(12): 1620-31. PubMed Abstract | Publisher Full Text | Free Full Text

77. Kievit RA, Brandmaier AM, Ziegler G, et al:: Developmental cognitive neuroscience using latent change score models: A tutorial and applications. Dev Cogn Neurosci. 2018; 33: 99-117. PubMed Abstract | Publisher Full Tex 


\section{Open Peer Review}

\section{Current Peer Review Status:}

\section{Version 1}

Reviewer Report 02 July 2019

https://doi.org/10.21956/wellcomeopenres.16620.r35724

(C) 2019 Hoorelbeke K. This is an open access peer review report distributed under the terms of the Creative Commons Attribution License, which permits unrestricted use, distribution, and reproduction in any medium, provided the original work is properly cited.

\section{Kristof Hoorelbeke}

Department of Experimental Clinical and Health Psychology, Ghent University, Ghent, Belgium

The presented design allows to address key questions of which the results will significantly expand our knowledge regarding the potential of cognitive control training as a preventive intervention for internalizing disorders in adolescents. An important advantage of this study is that it allows to explore long-term transfer effects. The manuscript is well-written, provides a clear overview of the design of the study and a transparent description of the to be conducted analyses. As such, I would like to compliment the researchers for the Open Science approach and fully support/endorse this timely study.

However, some questions remain (at least partially) unaddressed in the current manuscript:

1. The authors allow the participants to decide on which training task they complete following session three while suggesting them to conduct the dual $n$-back version. The rationale for this is not fully clear to me. In addition, this is likely to yield multiple training trajectories reflecting different difficulty levels (e.g., mixed single vs. double back tasks). This may form a confounding factor for some of the presented analyses. In addition, it complicates evaluation of training progress over time.

2. Related to this, the authors state that "opting to engage in a more challenging, but potentially more beneficial task is an index of self-regulation". From a developmental perspective the authors target a relatively wide age span (i.e., 11 - 19 years), inherently resulting in a sample showing strong heterogeneity in executive functions. As such, multiple other factors are likely to drive this choice (e.g., difficulty of the task, [reduced] maturation of executive control regions [due to a history of internalizing psychopathology], age appropriateness and attractiveness of the selected training procedures for the age group, etc., each of which may interact with other motivational factors). In addition, it would be interesting to also explicitly assess and model factors such as user engagement and task motivation.

3. Moreover, the introduction lacks a clear rationale for the age-related change hypothesis.

4. Although mixed yet encouraging findings have been presented regarding emotional 
transfer effects of cognitive control interventions, establishing cognitive transfer has been more challenging. In particular, cognitive transfer effects are often found to be task-specific. In a recent meta-analysis, $n$-back tasks have been classified as indicators of updating ability (Zetsche et al., (2018) $)^{1}$ ). To what extent can cognitive transfer effects be expected for other executive control functions such as inhibition and shifting ability, and how does this relate to emotional transfer effects?

5. Throughout the training procedure, the authors reinforce participants to conduct multiple sessions per day. What is the rationale for this, what do the authors consider as the 'optimal dose' for this type of cognitive control training and sample?

6. Developing a placebo/control training task is a challenging endeavor. However, the presented "placebo training" seems to be a non-adaptive task and as such does not allow to fully account for motivational effects of undergoing training given that the training procedure is adaptive. The authors could potentially control for this by adding a measure such as the Credibility and Expectancy Questionnaire at baseline and following training. Sadly, data-collection has already commenced for this study, which limits the modifications that can be made to the design to take into account the concerns raised by the reviewers of the presented protocol.

Nonetheless, I very much look forward for the results of this interesting study.

\section{References}

1. Zetsche U, Bürkner PC, Schulze L: Shedding light on the association between repetitive negative thinking and deficits in cognitive control - A meta-analysis.Clin Psychol Rev. 63: 56-65 PubMed Abstract | Publisher Full Text

Is the rationale for, and objectives of, the study clearly described? Partly

Is the study design appropriate for the research question? Yes

Are sufficient details of the methods provided to allow replication by others? Yes

Are the datasets clearly presented in a useable and accessible format? Not applicable

Competing Interests: No competing interests were disclosed.

Reviewer Expertise: Depression, cognitive vulnerability, cognitive control training, emotion regulation.

I confirm that I have read this submission and believe that I have an appropriate level of expertise to confirm that it is of an acceptable scientific standard. 
Author Response 23 Sep 2019

Susanne Schweizer, University College London, London, UK

Dear Dr Hoorelbeke,

Thank you for your helpful feedback on our protocol. Please find our responses below to the issues you raised in your report. Your original comments are printed in bold and our responses are copied below. Where appropriate we copied the amended/added sections from the manuscript in italics.

The authors allow the participants to decide on which training task they complete following session three while suggesting them to conduct the dual $n$-back version. The rationale for this is not fully clear to me. In addition, this is likely to yield multiple training trajectories reflecting different difficulty levels (e.g., mixed single vs. double back tasks). This may form a confounding factor for some of the presented analyses. In addition, it complicates evaluation of training progress over time.

Response: Please see our response to Dr Cohen.

Related to this, the authors state that "opting to engage in a more challenging, but potentially more beneficial task is an index of self-regulation". From a developmental perspective the authors target a relatively wide age span (i.e., 11 - 19 years), inherently resulting in a sample showing strong heterogeneity in executive functions. As such, multiple other factors are likely to drive this choice (e.g., difficulty of the task, [reduced] maturation of executive control regions [due to a history of internalizing psychopathology], age appropriateness and attractiveness of the selected training procedures for the age group, etc., each of which may interact with other motivational factors). In addition, it would be interesting to also explicitly assess and model factors such as user engagement and task motivation.

Response: We agree with the proposed age-related mechanisms, which is why we are explicitly investigating the moderating role of age on training effectiveness.

Unfortunately, we do not have other metrics of task motivation and user engagement than time spent training, which is why we cannot model these factors as proposed by the reviewer.

Moreover, the introduction lacks a clear rationale for the age-related change hypothesis.

Response: Please see our response to $\mathrm{Dr}$ Cohen.

Although mixed yet encouraging findings have been presented regarding emotional transfer effects of cognitive control interventions, establishing cognitive transfer has been more challenging. In particular, cognitive transfer effects are often found to be task-specific. In a recent meta-analysis, $n$-back tasks have been classified as indicators of updating ability (Zetsche et al., (2018)1). To what extent can cognitive transfer effects be expected for other executive control functions such as inhibition and 
shifting ability, and how does this relate to emotional transfer effects?

Response: This is an important question and one which has not been investigated in the context of affective control, which is why we are exploring the effectiveness of transfer to other facets of affective control. In a previous study we have shown transfer to affective inhibition as measured by the Stroop task included (see our response to Dr Cohen). The effects of the training on affective shifting, however, remain unexplored.

Throughout the training procedure, the authors reinforce participants to conduct multiple sessions per day. What is the rationale for this, what do the authors consider as the 'optimal dose' for this type of cognitive control training and sample?

Response: Given the relative lack of work in this age group and empirically driven "recommended dose" cannot be determined. As noted in our response to Dr Cohen the rationale for including the option to engage in multiple shorter training session is to maximize potential engagement of this age group with the training.

Developing a placebo/control training task is a challenging endeavor. However, the presented "placebo training" seems to be a non-adaptive task and as such does not allow to fully account for motivational effects of undergoing training given that the training procedure is adaptive. The authors could potentially control for this by adding a measure such as the Credibility and Expectancy Questionnaire at baseline and following training.

Response: We apologize for omitting this information in the previous version. The placebo training is also adaptive and we now explicitly state this in the training description:

"The initial trial includes 5 items per panel, the number of items per panel increases with participant's performance."

Competing Interests: NA

Reviewer Report 27 June 2019

https://doi.org/10.21956/wellcomeopenres.16620.r35725

(c) 2019 Owens M. This is an open access peer review report distributed under the terms of the Creative Commons Attribution License, which permits unrestricted use, distribution, and reproduction in any medium, provided the original work is properly cited.

\section{Max Owens}

University of South Florida St. Petersburg (USFSP), St. Petersburg, FL, USA

Summary: 
The proposed protocol outlines a double-blind and app-based training study seeking to improve affective cognitive control in adolescents (11-19 years). Training will consist of online practice on an adaptive and affective version of the $n$-back task, with participants allowed to choose between audio, visual and a dual-audio visual version across 11-days of a 14 day training period. In addition to the training task participants will complete a battery of mood and mental health questionnaires, as well as a battery of cognitive-affective tasks before and after training. The effects of training on changes in mood and mental health and task performance will be compared to a placebo control group completing one of three visual matching tasks consisting of shapes, words or faces. The author's will be testing four hypotheses predicting training related change in affective control on the $n$-back task, improvement on cognitive-affective tasks relative to the control group, that the benefits of training will decrease with age and that increased training effects on affective control will be associated with lower self-reported mood and mental health problems.

Critique:

The protocol is written with a very clear rationale and objectives. The methods are clearly described and seem appropriate for the objectives of the training, however please also include the maximum $n$-back level training participants can achieve. The study design seems largely appropriate as well, and has several strengths, including the use of an active control group, multiple outcome measures and follow-up periods. However, centrally, the reasoning behind allowing participants to choose which version of the training to take could be more clearly supported. If the research question is to compare the effectiveness of affective control training, why have participants potentially only completed less effective versions of the training (i.e. audio and visual only) versus the dual version? Additionally, given the adaptive nature of the tasks, increased improvement would seem to suggest a degree of self-regulation, so this construct could use a clearer operationalization.

Is the rationale for, and objectives of, the study clearly described?

Yes

Is the study design appropriate for the research question? Partly

Are sufficient details of the methods provided to allow replication by others? Partly

Are the datasets clearly presented in a useable and accessible format? Not applicable

Competing Interests: No competing interests were disclosed.

Reviewer Expertise: My research explores the nature, and remediation of, cognitive dysfunction in depression.

I confirm that I have read this submission and believe that I have an appropriate level of expertise to confirm that it is of an acceptable scientific standard. 


\title{
Author Response 23 Sep 2019
}

Susanne Schweizer, University College London, London, UK

Dear Dr Owens,

Thank you for your helpful feedback on our protocol. Please find our responses below to the issues you raised in your report. Your original comments are printed in bold and our responses are copied below. Where appropriate we copied the amended/added sections from the manuscript in italics.

However please also include the maximum n-back level training participants can achieve.

Response: The maximum level of $n$ back is not capped.

However, centrally, the reasoning behind allowing participants to choose which version of the training to take could be more clearly supported. If the research question is to compare the effectiveness of affective control training, why have participants potentially only completed less effective versions of the training (i.e. audio and visual only) versus the dual version?

Response: Please see our response to Dr Cohen.

Additionally, given the adaptive nature of the tasks, increased improvement would seem to suggest a degree of self-regulation, so this construct could use a clearer operationalization.

Response: The interactive role of improvement with our operationalization of selfregulation is an important consideration, which we will integrate into the interpretation of our results.

Competing Interests: NA

Reviewer Report 25 June 2019

https://doi.org/10.21956/wellcomeopenres.16620.r35727

(c) 2019 Cohen N. This is an open access peer review report distributed under the terms of the Creative Commons Attribution License, which permits unrestricted use, distribution, and reproduction in any medium, provided the original work is properly cited.

\author{
Noga Cohen \\ ${ }^{1}$ Department of Special Education, University of Haifa, Haifa, Israel \\ 2 The Edmond J. Safra Brain Research Center for the Study of Learning Disabilities, University of
}


Haifa, Haifa, Israel

In this report, Schweizer and colleagues propose a protocol for an app-based 14 day training procedure that is predicted to improve affective control, mental health, emotion regulation and self-regulation among youths (11-19 years).

The authors propose an important and timely study, designed to test whether affective control training can be beneficial for youths. The design proposed in this protocol is based on the authors' prior work showing improved emotion regulation following an affective control (working memory) training.

The paper is well written and the proposed study is expected to advance our understanding on the mechanisms involved in emotion regulation and behavioral deficits among youths, as well as open new avenues for treatment. My comments are mainly related to clarification of tasks-related aspects.

What is the rational for allowing participants to choose the version of the task on days 4-14?

Moreover, by reading the "The present study" paragraph it is not clear whether participants in the control group (placebo training) will receive the same instructions - allowing them to choose and prompting them to prioritize one of the versions over the other two versions. Although this information is mentioned later in the paper, I suggest referring to this issue already in "the present study" paragraph.

The authors plan to include both positive and negative stimuli in the affective control training. Are there studies showing beneficial outcomes for this type of training when positive stimuli are used (besides work on eating and addiction with the go/no-go and stop signal tasks)? Can the authors elaborate a bit more about their decision to include positive stimuli in the training?

Can the authors say something about the similarity/difference of the affective control and placebo training in regard to difficulty? Are accuracy rate and mean reaction time more or less similar in the working memory and perceptual tasks? It can be nice to show that training outcomes are not modulated by task difficulty (therefore strengthening the authors' notion that the outcomes are specific to improvement in affective control).

In line with the previous comment, assessing the links between training outcomes in terms of the cognitive/affective control tasks and the self-report measures can be highly valuable to the understanding of the processes by which the training influence emotion regulation, mood, as well as psychological and behavioral difficulties.

Pre/post changes in inhibition will be assessed using a modified Stroop task. Did the authors consider using a more "classic" inhibition task (e.g., go/no-go, stop signal)? Why Stroop?

In the predictions section the authors write that they plan to test improvement in affective control by using an affective $n$-back task (which is different from the training task). The authors mention later in the paper that this task will be administered before and after the training. I suggest mentioning this before the "Methods" section because when I read the predictions section it was not clear to me. In addition, why not use the training task itself to assess changes in affective 
control? Is it because participants choose the version of the task on days 4-14?

The authors predict that the benefits of AC-Training will decrease with age (age-related change hypothesis). I advise the authors to relate to this issue in the introduction. Is this prediction based on prior literature?

The authors write that participants will complete 14 days of training within a four-week period. Moreover, they mention that there will be no limit on the number of training sessions participants can complete during a day. Do they mean that during the 4-week period there are 14 specific days in which participants can do the training? On a training day, can participants do a 2-minute task 5 times to complete the session? I find it hard to follow the training procedure. Moreover, what is the rational for enabling participants to choose the number of training sessions?

On each training session, participants will be asked "Are you trying to change the way you feel right now?" and could choose a strategy - distraction, problem-solving, behavioural activation, reappraisal, avoidance, social support, acceptance or other. I guess that these strategies will be explained at the pre-training session. Can the authors relate to this? They can do that in the "extended data" document if they prefer not to include these details in the main paper.

What software will be used to program the training apps, questionnaires, and pre/post tasks? Will these apps/tasks be available for other researchers? Are the apps compatible with both Android and Apple smartphone devices?

The authors mention that "total training time will allow us to explore dose-response relationships". However, this time may also reflect motivation/choice-related factors as participants choose how much time they wish to spend on the task.

Is the rationale for, and objectives of, the study clearly described?

Yes

Is the study design appropriate for the research question?

Yes

Are sufficient details of the methods provided to allow replication by others? Partly

Are the datasets clearly presented in a useable and accessible format? Not applicable

Competing Interests: No competing interests were disclosed.

Reviewer Expertise: Emotion regulation, attention, cognitive control, training

I confirm that I have read this submission and believe that I have an appropriate level of expertise to confirm that it is of an acceptable scientific standard, however I have significant reservations, as outlined above. 
Author Response 23 Sep 2019

Susanne Schweizer, University College London, London, UK

Dear Dr Cohen,

Thank you for your helpful feedback on our protocol. Please find our responses below to the issues you raised in your report. Your original comments are printed in bold and our responses are copied below. Where appropriate we copied the amended/added sections from the manuscript in italics.

What is the rational for allowing participants to choose the version of the task on days 4-14?

Response: We are interested in exploring the role of self-regulation in cognitive training. Providing a rationale for one training $(C)$ being associated with superior benefits compared to $A$ and $B$ should motivate individuals to select $C$ more often. However, $C$ is more cognitively demanding, therefore requiring self-regulation to engage in this task over the others for potential future benefits. We now elaborate on this further in the manuscript.

"On the first three days of the 14-day training programme, participants will train on one version each day. On days 4-14, participants are free to select any or all of the training versions. However, both training groups will be provided with a rationale suggesting that training on version $C$ is likely to confer more benefits than the other two versions. By providing a rationale for one training being associated with superior benefits compared to others, participants should be motivated to select $C$ over the other two training versions. However, $C$ is more cognitively demanding, therefore requiring self-regulation to engage in this task over the others for potential future benefits. We hypothesise that opting to engage in a more challenging but potentially more beneficial task is an index of self-regulation, which has been shown to be associated with mental health across the lifespan ${ }^{36}$. This will allow us to explore the role of self-regulation in cognitive training and any effects on mental health outcomes."

Moreover, by reading the "The present study" paragraph it is not clear whether participants in the control group (placebo training) will receive the same instructions allowing them to choose and prompting them to prioritize one of the versions over the other two versions. Although this information is mentioned later in the paper, I suggest referring to this issue already in "the present study" paragraph.

Response: We have modified the section accordingly (see above).

The authors plan to include both positive and negative stimuli in the affective control training. Are there studies showing beneficial outcomes for this type of training when positive stimuli are used (besides work on eating and addiction with the go/no-go and stop signal tasks)? Can the authors elaborate a bit more about their decision to include positive stimuli in the training?

Response: We opted to train affective control across valences because aberrant reward processing is a core characteristic of mood disorders. Similarly, we have recently shown in a 
meta-analyses that affective control measured with working memory tasks such as the training task is equally impaired in individuals with mental health problems across valences. We now elaborate on this further in the background section:

"The rationale for including stimuli of different valences is that mental health problems can be characterised by difficulties disengaging from negative material ${ }^{37}$, avoidance of negative (e.g., threatening) information ${ }^{38}$ or aberrant processing of positive information ${ }^{39}$. Moreover, we have recently shown in a meta-analysis that, in individuals with mental health problems, affective control, measured with working memory tasks such as the training task, is similar across positive and negative valence?."

Can the authors say something about the similarity/difference of the affective control and placebo training in regard to difficulty? Are accuracy rate and mean reaction time more or less similar in the working memory and perceptual tasks? It can be nice to show that training outcomes are not modulated by task difficulty (therefore strengthening the authors' notion that the outcomes are specific to improvement in affective control).

Response: While the placebo task is engaging and challenging it cannot be directly compared to affective control training task in terms of either accuracy or reaction time as the former is a self-paced search task. We will consider the potentially confounding effect of task difficulty in our interpretation of our findings.

In line with the previous comment, assessing the links between training outcomes in terms of the cognitive/affective control tasks and the self-report measures can be highly valuable to the understanding of the processes by which the training influence emotion regulation, mood, as well as psychological and behavioral difficulties. Pre/post changes in inhibition will be assessed using a modified Stroop task. Did the authors consider using a more "classic" inhibition task (e.g., go/no-go, stop signal)? Why Stroop?

Response: We have included the Stroop task to replicate findings from the adult literature, which have shown the affective control performance to improve affective Stroop performance (Schweizer et al., PLOS ONE, 2011).

In the predictions section the authors write that they plan to test improvement in affective control by using an affective $n$-back task (which is different from the training task). The authors mention later in the paper that this task will be administered before and after the training. I suggest mentioning this before the "Methods" section because when I read the predictions section it was not clear to me. In addition, why not use the training task itself to assess changes in affective control? Is it because participants choose the version of the task on days 4-14?

Response: We have removed the reference to the different versions as we appreciate the confusion this caused. As noted in the methods, the task is the same but includes nontrained stimuli sets as well as a different progression and termination rule. That is, each level of $n$ is presented twice. The task terminates once both blocks at a given level are 
completed incorrectly.

The authors predict that the benefits of AC-Training will decrease with age (agerelated change hypothesis). I advise the authors to relate to this issue in the introduction. Is this prediction based on prior literature?

Reponses: We have modified the hypothesis to be non-directional as we agree that there is not sufficient evidence to support a directional hypothesis. We now also refer the agerelated hypothesis earlier stating:

"Specifically, we will explore whether AffeCT improves adolescents' mental health and emotion regulation capacity and whether the magnitude of these improvements differs as a function of age.

To investigate the potential of AffeCT in adolescents, the current study will include 200 adolescents (11-19 years). Including this age range will allow us to investigate the potential agerelated differences in the effectiveness of training, as shown in studies using cool cognitive training paradigms 76 ."

The authors write that participants will complete 14 days of training within a fourweek period. Moreover, they mention that there will be no limit on the number of training sessions participants can complete during a day. Do they mean that during the 4-week period there are 14 specific days in which participants can do the training? On a training day, can participants do a 2-minute task 5 times to complete the session? I find it hard to follow the training procedure. Moreover, what is the rational for enabling participants to choose the number of training sessions?

Response: A full training regime is considered 14 days but participants can opt to train for more days. There will be no limit on the number of training sessions they can complete during a day. A full training session takes between 20-30 minutes but participants have the option to end the training after 10 minutes. Anything less than 10 minutes will not be counted as a training session. The reason for allowing participants to stop the training after 10 minutes is to increase engagement with the training in this age group. Allowing for multiple training sessions in a day enhances the possibility of maximizing training time in a sample who is likely not to engage in an activity for extended amounts of time but who might be motivated by the monetary compensation to engage in a task repeatedly.

In the methods we note:

"[...] participants will be given the option to end the training any time from 10 mins onward. The full training session will take between 20-30 mins depending on the level achieved. There will be no limit on the number of training sessions they can complete during a day. Training sessions that are less than 10 mins will not be considered as full training sessions, and will not be included in the analyses, nor will participants be compensated for these sessions."

On each training session, participants will be asked "Are you trying to change the way you feel right now?" and could choose a strategy - distraction, problem-solving, behavioural activation, reappraisal, avoidance, social support, acceptance or other. I guess that these strategies will be explained at the pre-training session. Can the 
authors relate to this? They can do that in the "extended data" document if they prefer not to include these details in the main paper.

Response: Each strategy is described in the answer options. The strategy names in brackets are not included in the drop down menu.

Are you trying to change the way you feel right now?

No

Yes, by distracting myself. (Distraction)

Yes, by dealing with my problem. (Problem-solving)

Yes, by doing something fun. (Behavioural activation)

Yes, by thinking about the problem differently. (Reappraisal)

Yes, by avoiding the problem. (Avoidance)

Yes, by spending time with family/friends. (Social support)

Yes, by accepting the situation. (Acceptance)

Other

What software will be used to program the training apps, questionnaires, and pre/post tasks? Will these apps/tasks be available for other researchers? Are the apps compatible with both Android and Apple smartphone devices?

Response: Once the results from this study and other ongoing studies using the apps are published the apps will be made available to other researchers. The apps are compatible with both Android and Apple devices. The app and tasks are coded in javascript and HTML5. To get them to run on the mobile devices we are using a software package called Cordova which converts the Javascript and HTML5 into the native languages of the devices.

The authors mention that "total training time will allow us to explore dose-response relationships". However, this time may also reflect motivation/choice-related factors as participants choose how much time they wish to spend on the task.

Response: This is an important consideration, which we will incorporate into the interpretation of our results.

Competing Interests: NA

Reviewer Report 25 June 2019

https://doi.org/10.21956/wellcomeopenres.16620.r35726

(c) 2019 Derakhshan N. This is an open access peer review report distributed under the terms of the Creative Commons Attribution License, which permits unrestricted use, distribution, and reproduction in any medium, provided the original work is properly cited.

Nazanin Derakhshan 
Department of Psychological Sciences, Birkbeck, University of London, London, UK

This is an excellent and timely study with much potential for impact on adolescent mental health. The aim is to improve affective control and hopefully emotion regulation strategies in young and older adolescents using affective control training, a paradigm used by the first author before with good results.

The study is longitudinal in nature which is to be commended. The longitudinal effects of AC training will be studied on self-report measures of mood and emotional vulnerability and emotion regulation as well as performance measures of executive control functioning and working memory performance, which are crucial to investigate.

The methods of analysis addressing each of the predictions are sound and adequate, clearly explained and solid.

The characteristics of the AC training and the Placebo (P) training are well defined and easy to follow. I have a few comments/remarks that the authors might want to consider:

First, would there be a problem in letting the participants 'choose' the single (auditory or visual) or the dual $n$-back training after day 3? I would assume that the single $n$-back can be less demanding on working memory resources than the dual $n$-back which can be more engaging for the participant. In this way, how can we control for possible confounds as a matter of choice of training? How can we compare the efficacy of the single to the dual, which I am guessing is a noteworthy comparison to do anyway, but won't be possible given the unsystematic manipulation if any.

Second, it isn't clear if participants will do the training at home or at school if they are using an app? There is a mention of an app and that the study will run in schools - in form of a group testing session? Or individually?

I fully support this study and I hope that the findings can pave the way towards promoting as well as sustaining better mental health in a population in most need of it.

Is the rationale for, and objectives of, the study clearly described?

Yes

Is the study design appropriate for the research question?

Yes

Are sufficient details of the methods provided to allow replication by others?

Yes

Are the datasets clearly presented in a useable and accessible format?

Yes

Competing Interests: No competing interests were disclosed. 
Reviewer Expertise: Affective and Cognitive Neuroscience, Neurocognitive mechanisms of emotional vulnerability and resilience in anxiety and depression (adults and adolescents) and breast cancer survivorship.

I confirm that I have read this submission and believe that I have an appropriate level of expertise to confirm that it is of an acceptable scientific standard.

Author Response 23 Sep 2019

Susanne Schweizer, University College London, London, UK

Dear Professor Derakshan,

Thank you for your helpful feedback on our protocol. Please find our responses below to the issues you raised in your report. Your original comments are printed in bold and our responses are copied below. Where appropriate we copied the amended/added sections from the manuscript in italics.

First, would there be a problem in letting the participants 'choose' the single (auditory or visual) or the dual n-back training after day 3 ? I would assume that the single $n$ back can be less demanding on working memory resources than the dual $n$-back which can be more engaging for the participant. In this way, how can we control for possible confounds as a matter of choice of training? How can we compare the efficacy of the single to the dual, which I am guessing is a noteworthy comparison to do anyway, but won't be possible given the unsystematic manipulation if any.

Response: We agree that the effects of the training are likely to vary as a function of the training version selected. As suggested by Professor Derakshan we will look at the effectiveness of training as a function of the training version selected. Specifically, we will look at the effects of time trained on each version on our outcomes of interest. We will additionally also run a comparison of time trained on the dual version versus time trained on the single versions. We now explicitly state this in the analysis section:

"We will additionally investigate the effect of time spent on each training version separately on the outcome of interest and compare the effect of time spent on the single versions versus time spent on the dual version."

Second, it isn't clear if participants will do the training at home or at school if they are using an app? There is a mention of an app and that the study will run in schools - in form of a group testing session? Or individually?

Response: Participants will do the training in their own time, on their own devices (any device that supports mobile apps). We now specified this further in the methods.

"Participants complete a pre-training assessment, followed by 14 days of training within a fourweek period. The training will be completed by the participants on their own devices (any device that supports mobile apps) outside of school time." 
Competing Interests: NA 